\title{
Feasibility and efficacy of double over-the-scope clipping for colonic iatrogenic perforation
}

Paola Soriani, MD, Carmelo Barbera, MD, Vincenzo Giorgio Mirante, MD, Lucia Miglioli, MD, Mauro Manno, MD

Colorectal iatrogenic perforation is a rare adverse event in diagnostic colonoscopy, occurring in a range of $0.03 \%$ to $0.8 \%$ of cases. Risk factors include endoscopist's inexperience, female gender, pericolic adhesions, inflammatory colonic diseases, severe diverticular disease, and weakened colonic-wall tissues because of older age. ${ }^{1}$

The over-the-scope clip (OTSC, Ovesco Endoscopy $\mathrm{GmbH}$, Tübingen, Germany) is a useful tool, recommended as first-line endoscopic treatment for endoscopic acute iatrogenic perforation, ${ }^{1}$ which can help to avoid emergency surgical repair. ${ }^{2}$ However, the deployment of an OTSC to completely close the defect may be challenging in some cases because of the size, the position of the hole (ie, sigmoid-rectal junction), and the presence of other endoscopic devices (ie, through-thescope clip or OTSC). To the best of our knowledge, the deployment of 2 adjacent OTSCs has not been described in the medical literature.

Here we report the case of an 89-year-old woman referred to our unit for colonoscopy (CFHQ190L; Olympus Co, Tokyo, Japan) because of a positive hemoccult test result and anemia. Because of pericolic adhesions in a

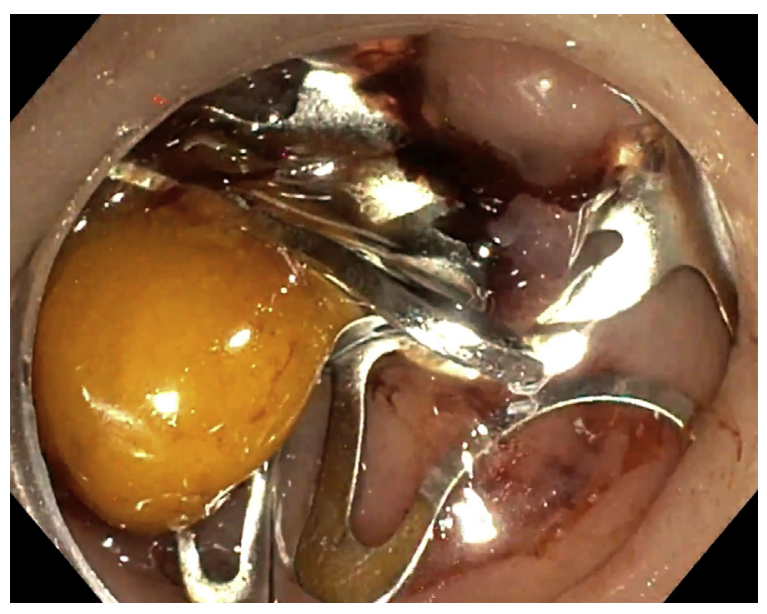

Figure 1. Endoscopic appearance after deployment of the second over-the-scope clip, showing no stenosis of the lumen. case of severe diverticular disease, a perforation of about 20 to $25 \mathrm{~mm}$ occurred in the sigmoid-rectal junction.

After a nasogastric tube and a Verres needle were positioned to favor abdominal decompression, a preliminary deployment of an OTSC (11/6 mm traumatic type) with suction technique did not achieve the complete seal of the defect. Indeed, another analogous OTSC was deployed with the suction technique tightly adjacent to the first one, obtaining total closure of the leakage (Fig. 1) as confirmed by the injection of contrast medium (Fig. 2) and subsequent CT scan (Video 1, available online at www. VideoGIE.org).

The entire procedure was performed with the patient under deep sedation with the use of $\mathrm{CO}_{2}$ insufflation. No further adverse events occurred, broad-spectrum antibiotics were administered, and the asymptomatic patient was discharged 1 week later.

In conclusion, even though the procedure is challenging, the closely positioned deployment of 2 OTSCs is feasible and effective for treating iatrogenic perforations. Furthermore, it can help to avoid later adverse events or

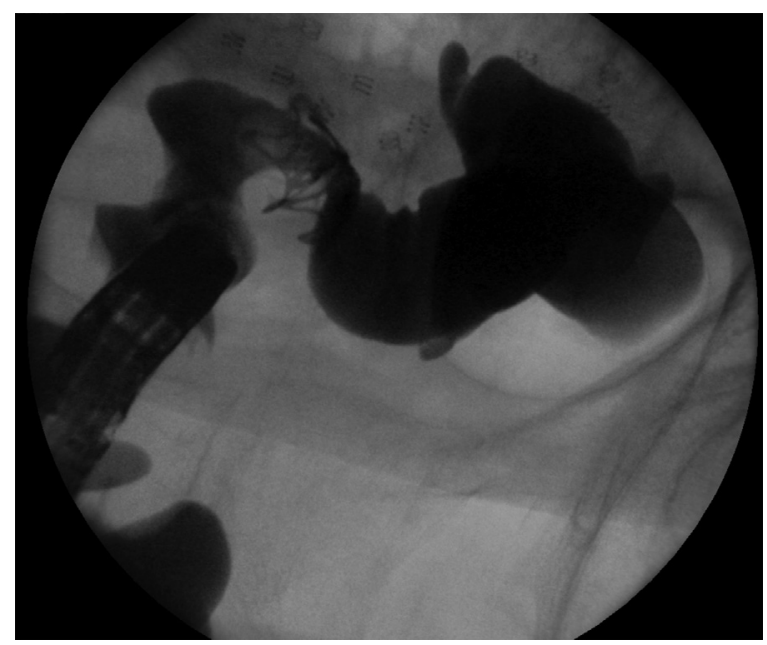

Figure 2. Complete closure of leakage after injection of contrast medium. 
surgical repair, especially in patients at high risk for anesthesia.

\section{DISCLOSURE}

All authors disclosed no financial relationships relevant to this publication.

\section{REFERENCES}

1. Paspatis GA, Dumonceau JM, Barthet M, et al. Diagnosis and management of iatrogenic endoscopic perforations: European Society of
Gastrointestinal Endoscopy (ESGE) Position Statement. Endoscopy 2014;46:693-711.

2. Donatelli G, Cereatti F, Dhumane P, et al. Closure of gastrointestinal defects with Ovesco clip: long-term results and clinical Implications. Ther Adv Gastroenterol 2016;9:713-21.

Digestive Endoscopy Unit, Azienda USL Modena, Ramazzini Hospital, Carpi, Italy.

Copyright (c) 2018 American Society for Gastrointestinal Endoscopy. Published by Elsevier Inc. This is an open access article under the CC BYNC-ND license (http://creativecommons.org/licenses/by-nc-nd/4.0/).

https://doi.org/10.1016/j.vgie.2017.10.009

\section{Facebook}

Follow VideoGIE on Facebook to receive the latest news, updates, and article links. Visit https://www.facebook.com/videogiejournal/ and keep checking back to view our most recent posts. 